\title{
Dynamic Repair and Robust Optimization of Complex Networks
}

\author{
Pengtao Zhang, Peng Bai, Chaoqi Fu $(\mathbb{D}$, and Shanshan Li
}

Air Force Engineering University, Xi'an 710038, China

Correspondence should be addressed to Chaoqi Fu; fuchaoqi2011@163.com

Received 12 February 2020; Accepted 7 May 2020; Published 31 May 2020

Academic Editor: Xiangyu Meng

Copyright (c) 2020 Pengtao Zhang et al. This is an open access article distributed under the Creative Commons Attribution License, which permits unrestricted use, distribution, and reproduction in any medium, provided the original work is properly cited.

Network repair is indispensable for maintaining network security. Conventional static repair is relatively inefficient. In this study, by considering the energy transfer between nodes, a dynamic repair model was established. The fundamental reason for the secondary failure of repaired nodes during the dynamic repair process is the coupling structure of failure networks. A dynamic repair strategy was proposed that can effectively prevent the secondary failure of repair nodes influenced by energy during repair and can cause the redundant capacity of repair nodes to be used reasonably. By turning off the energy transfer function of the link to control the excessive flow of energy into the repair node to avoid the occurrence of secondary failure; on the other hand, by sharing part of the load of the failure node, realize the rational use of the redundant capacity of the repair node to reduce the impact of the failure node on the overall function of the network. The proposed strategy mitigated the effect of failure nodes on network functions and substantially improved the recovery efficiency of network functions. Furthermore, redundant edges, behaving as energy redundant links in a network structure, can considerably improve the robustness of the network by optimizing the removal of redundant edges. Dynamic repair is not only an efficient repair method but also a highly flexible choice for network repair.

\section{Introduction}

With the rapid development of computer technology, complex network theory has been widely studied [1-8]. Many favorable achievements have been obtained in different areas of tangible objects in a Euclidean space (electric power grids [2], transportation networks [3], and the Internet [4]) and in entities defined in an abstract space (communication relationships [5], management networks [6], links between worlds [7], and other crucial areas [8]). A complex network is a theoretical method to study complex systems as a whole. By abstracting the units in a complex system as nodes and the connections between the units as links and then using a graph theory to network the complex system, we can study the related problems of the complex system in terms of topology, such as node importance [9], degree correlations [10], network robustness [11], and network invulnerability [12]. However, the phenomenon of cascading failure in disasters such as the North American blackout cannot be explained from the topology very well. Cascading failure takes place under certain initial disturbance, leading to an avalanche of overloads on the other nodes. If the energy interaction between the units is also considered, the hidden dangers and deficiencies of the energy interaction between units in the entire complex system can be clearly understood [13]. Research on complex networks is helpful for optimizing the design of network structures [14, 15]. However, many real-life complex systems often have many defects and deficiencies due to social progress and the limitations of the initial design $[16,17]$. Due to various reasons such as technological development, economic constraints, and policy orientation, we cannot timely reconstruct these existing networks on a large scale and can only gradually improve them step by step.

Under this premise, studying how to effectively repair the network after a breakdown is valuable. After years of research, we gained a deeper understanding of recovering networks. Numerous repair strategies have been proposed [18-22]. Chi et al. $[16,18]$ studied the stability of complex networks under the evolution of attack and repair. Farr et al. [19] studied optimal networks on regular lattices and 
established a repairable network structure. Sun and Zeng [20] studied target recovery in complex networks, and appropriate repair sequence can be determined using their proposed hybrid recovery method. Li et al. [21] established a model of cascading failures with an emergency recovery mechanism, which considerably improved the invulnerability of the studied network. Additionally, Majdandzic et al. [22] studied the spontaneous recovery in dynamic networks. However, few studies on network repair have considered the effects of energy transfer between nodes. The main reason for the collapse of many networks is the cascading failure caused by energy redistribution. The neglect of energy transfer during repair causes the repair of the network to degenerate into static repair, isolating the demand and effect of failure nodes on the network, resulting in a relatively inefficient repair model.

In this paper, we propose a dynamic repair model that considers the effect of energy transfer caused by failed nodes on network recovery. Moreover, a dynamic repair strategy is proposed that effectively prevents the secondary failure of repaired nodes during the repair process. The reasonable use of redundant capacity of repaired nodes can share some of functional loads of failed nodes, reduce the effect of failed nodes on the network function recovery, and considerably improve the efficiency of the network functional recovery. Furthermore, two robust optimization methods are proposed to improve the robustness of the network.

\section{Dynamic Repair Modeling}

A graph was established as $G=(V, E)$, where $V=\left\{v_{1}, v_{2}, L, v_{n}\right\}$ is a set of nodes and $E=\left\{e_{1}, e_{2}, L, e_{n}\right\}$ is a set of edges.

The real network can be divided into two overlapping networks, as illustrated in Figure 1. One is a foundational network with undirected and unweighted parameters; this represents the physical structure of the network, such as roads and blood vessels. The other is a directional weighting network; this so-termed functional network represents functions performed using the foundational network, such as vehicles traveling on roads and blood flowing in blood vessels. Collectively, this is referred to as energy.

An avalanche effect cannot be explained by simply studying the network topology. The cascading failure caused by the improper chaos and mismatch of energy is considered to be the main cause of network collapse. When one node fails, the energy is redistributed to other functioning nodes. When the recalculated energy exceeds the redundant capacity, the node fails and the redistribution of the energy leads to a new round of failure, causing a large-scale network collapse [23]. We selected a model defined by Yang et al. [14], namely, $C_{i}=\alpha+\beta L_{i}=L_{i}+\Delta C_{i}$, where $\alpha$ and $\beta$ are tolerance coefficients and $\Delta C_{i}$ is the redundant capacity; the initial load of the node is correlated with its link degree $k_{i}$ as $L_{i}=k_{i}^{\theta}$, where $\theta$ is the load parameter [24]. When the network was attacked, the energy of the failed node $i$ was reassigned to its adjacent nodes according to the following equation:

$$
\Delta L_{i j}=L_{i} \times \frac{L_{j}}{\sum_{k \in \Gamma_{i}} L_{k}},
$$

where $\Delta L_{i j}$ is the redistribution energy from the failed node $i$ to the functioning node $j$ and $\Gamma_{i}$ is a set of functioning nodes adjacent to the failed node $i$.

The energy disorder was caused by changes in the network structure. Therefore, energy was disrupted when the network was destroyed and when it was repaired. Unlike static repair models, dynamic repair models consider the effect of failed nodes on network function recovery. The repaired node can immediately perform its function, and the redundant capacity of repaired nodes can share some of the network functions of failed nodes; this can mitigate the effect of failed nodes on the whole network. This mechanism substantially improved the efficiency of network function recovery. According to equation (1), the load redistribution of repair rules [25] are as follows:

$$
\Delta L_{j i}=L_{j} \times \frac{L_{i}}{\sum_{k \in \Gamma_{j}} L_{k}},
$$

where $\Gamma_{j}$ is the node adjacent to the failed node $j$. Therefore, the total redistribution load of the repaired node $i$ is $\Delta L_{i}=\sum_{j \in \Pi_{i}} \Delta L_{j i}$, where $\Pi_{i}$ is the set of failed nodes adjacent to node $i$. When $\Delta L_{i}>\Delta C_{i}$, node $i$ failed again.

\section{Dynamic Repair Strategy and Algorithm}

In the dynamic repair model, the process of repairing network involved energy transfer between nodes. Although secondary failure can occur, an appropriate repair strategy can reduce the risk of failure and restore network functions more effectively. Each node has different tolerance levels toward energy shocks and each edge carries different energy levels. All the factors caused dynamic repair to become more complex and polytropic.

Figure 2 presents the classification of the network structure. The number on the node was $\Delta C$. The number on the directed edge $e_{i j}$ indicated the energy transfer from node $i$ to node $j$. Figure 2(a) is an uncoupled structure that can be repaired using a special sequence: $A-B-C$ or $A-C-B$. Node $A$ must be repaired before node $B$ and $C$. When node $A$ was repaired, nodes $B$ and $C$ were no longer affected by the redistributed energy from node $A$. Therefore, the total redistribution load of nodes $B$ and $C$ did not exceed their redundant capacity, and nodes $B$ and $C$ were repaired without the secondary failure. Therefore, the nodes in the uncoupled structure were repaired one by one by following a repair sequence. Subsequently, some more rigorous and complicated structures remained, as shown in Figure 2(b), which were termed coupled structures. When any of the nodes in the coupled structure were repaired, a secondary failure occurred because the redistributed energy was transferred from other nodes to the structure that exceeded the redundant capacity of the repaired node. For node $D$, the redistributed load from nodes $E$ and $G$ exceeded its redundant capacity, regardless of the energy effects of nodes $B$ and $C$ on it $\left(\Delta C_{D}<\Delta L_{E D}+\Delta L_{G D}\right)$. Therefore, 


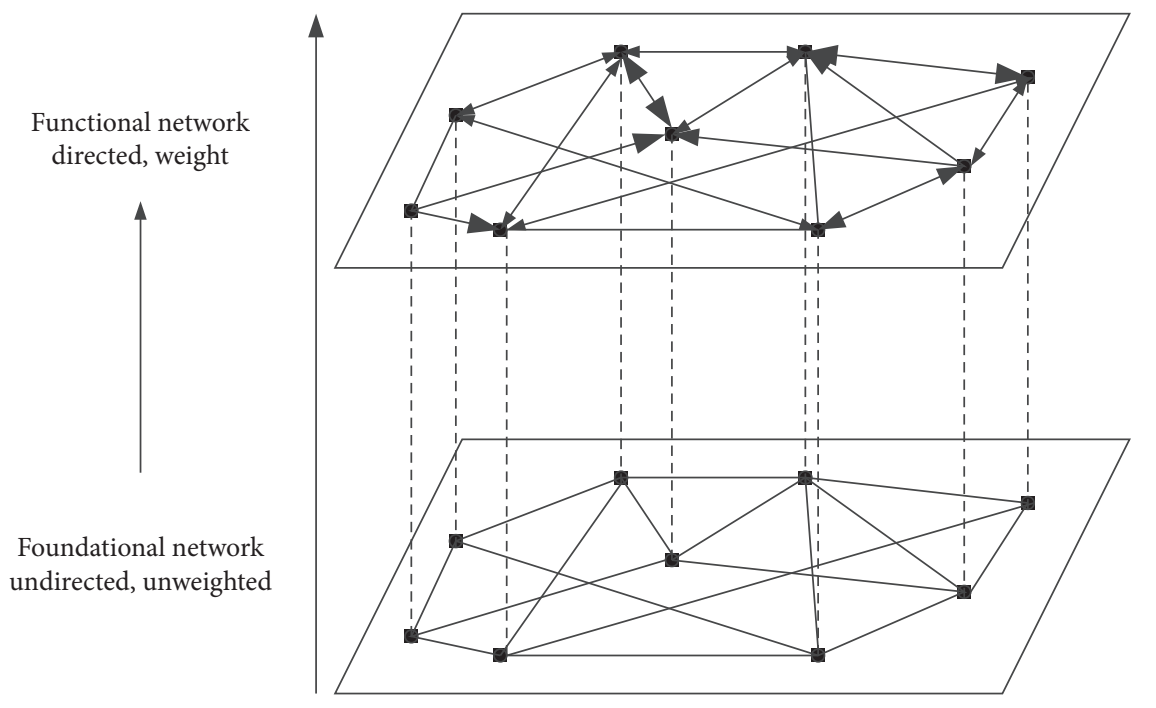

Figure 1: Uncoupled structure.

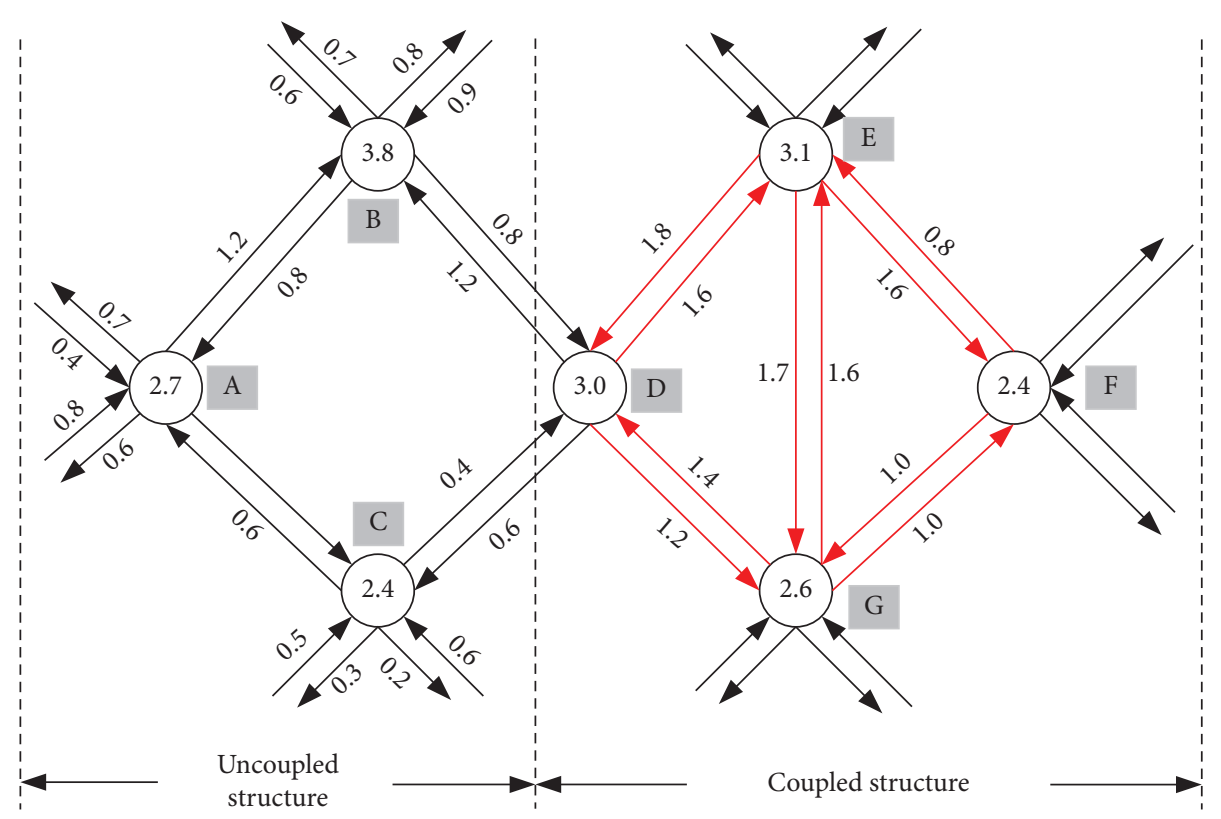

(a)

(b)

FIgURE 2: Classification of network structure.

additional measures must be taken to break the coupling relationship before repair. Maintenance personnel were involved in the repair process of damaged network; therefore, the function of related links was temporarily shutdown to reduce the energy transfer from these links to the repaired nodes. When the condition $\Delta L<\Delta C$ was satisfied, failed nodes were repaired without the risk of a secondary failure. The closing link $e_{E D}$ led to several different repair sequences, such as $D-E-G-F$ and $E-D-B-F$ $G$. However, selecting the correct link in the coupled structure for temporary shutdown was crucial. For example, if the closed link was $e_{E F}$ or $e_{F G}$, the nodes $E, D$, and $G$ still could not be repaired. Therefore, we proposed the following repair strategy. By cutting off the minimum number of link functions, the coupling structures were degraded into uncoupled structures. Then, the uncoupled structures were repaired using an appropriate repair sequence to recover the damaged network. The steps of the dynamic repair strategy are shown in Figure 3.

Step 1: construct a failed network.

Failed nodes and their relationships can be extracted to construct a failed network. Consider $S$ is the number of failed nodes and $\widehat{k}$ is the degree centrality of new nodes (calculated in the failed network).

Step 2: filter the coupling structures. 


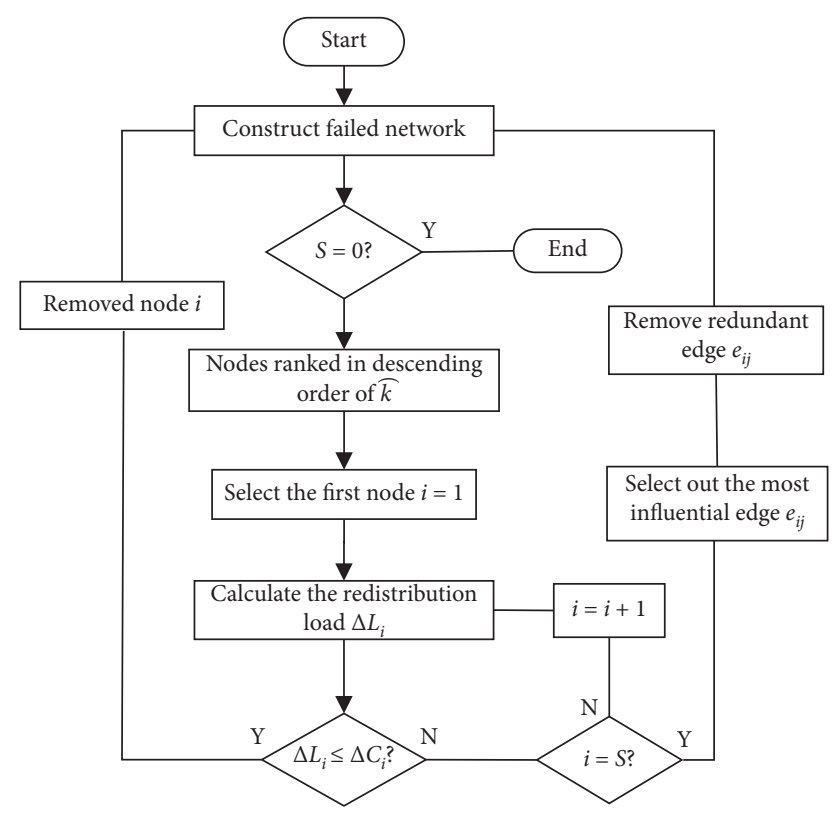

Figure 3: Algorithm flowchart.

First, calculate the redistribution load of each node at a time and repair node $i$ when it satisfies $\Delta L_{i}<\Delta C_{i}$. Subsequently, return to step 1 to reconstruct a new failed network if $S \neq 0$. However, if the redistributed load of all the nodes exceeds the redundant capacity of the node, only coupled structures are similar to those presented in Figure 2 in the failed network. Then, proceed to step 3 .

Step 3: filter the redundant edge and remove it.

Coupled structures are the key to determining whether failed nodes can be repaired without secondary failure. The damage of coupling structures can be divided into two stages: (1) closing the link function of energy transfer affects the load redistribution of received nodes at both ends; (2) if the function of the link is closed in stage (1) and some nodes can be repaired without secondary failure, the effect of repaired nodes on neighboring nodes is similar to that described in stage (1). Therefore, the damage assessment of closed links is a superposition of the two stages and has a greater effect. We designed the following rules, called "redundant edges," to filter closed links.

(1) Filter out all links that cause nodes at both ends to satisfy $\Delta L_{i}<\Delta C_{i}$ and then close the functions of the link with the largest sum of the degree of nodes at both ends. This may cause severe damage to coupled structures.

(2) If no link satisfies rule (1), the filtering criteria are degraded to have a node at both ends of the link, satisfying the condition $\Delta L_{i}<\Delta C_{i}$. Close the link with the node satisfying the condition $\Delta L_{i}<\Delta C_{i}$ and the maximum degree $k$.

(3) If neither of the aforementioned two criteria are met, the closed link is considered to be the one with maximum transfer energy. The higher the energy $e_{i j}+e_{j i}$ is, the greater is the damage to coupling structures. Therefore, subsequently, satisfying the first two rules during screening becomes easier, and the screening process forms a closed cycle.

If one of the three rules is satisfied, close the link function, remove it from the failed network, and then return to Step 1 to reconstruct the new failed network.

\section{Simulation Verification and Analysis}

A scale-free network (SFN) [26] was constructed to verify the effectiveness of the dynamic repair strategy. An SFN is a growing complex network with a network average degree $k$ of approximately 4 . The initial scale had 15 nodes, and the number of final nodes was $N=1000$. A study on the load model indicated that networks exhibited the most robustness [24] under the condition of $\theta=1$. One of the tolerance coefficients was fixed, and the other one, $\alpha$ (or $\beta$ ), was variable. First, the node with the maximum degree was attacked, which caused cascading failure in the network. Then, the damaged network was repaired using the proposed strategy. The simulation results are presented in Figure 4.

Figure 4(a) presents the scale of redundant edges observed in scale-free networks; each value is the average of results obtained under 20 independent SFN networks. The failed nodes (FNs) represent the number of failed nodes in the damaged network. The closed link (CL) represents the number of redundant edges that are closed functions. The simulation results revealed that the $\mathrm{FNs}$ can be repaired without the risk of secondary failure by closing a small number of redundant edges at an appropriate time and by using an appropriate repair sequence. After all of the affected links were compared, the average number of CLs was 55.6, considerably less than 1323.9, and as the $\alpha$ decreased, the number of FNs became less than that of CL, indicating that the efficient recovery of the network functionality can be achieved at a low cost in dynamic repair process (Figure $4(\mathrm{a})(\mathrm{A}))$. Similar results were obtained for the change in $\beta$ (Figure 4(a)(B)). Figure 4(b) presents the simulation results under the real-life network and scientific cooperation network (SCN) [27]. The maximum connectivity in the SCN is defined using the failed network, total number of nodes $(N=379)$, and number of edges $(E=914)$. Figure 4(b) indicates that the number of redundant edges in the dynamic repair process was negatively correlated with tolerance coefficients $\alpha$ (Figure $4(\mathrm{~b})(\mathrm{A})$ ) and $\beta$ (Figure 4(b)(B)). The smaller the values of $\alpha$ and $\beta$ were, the smaller was the redundant capacity of nodes and the more severe was the restriction on the energy transfer of each edge. Therefore, more redundant edges must be temporarily removed from the energy transfer function. However, compared with the static model, the dynamic repair model effectively used the redundant capacity of nodes so that repaired nodes can reasonably share the partial load of FNs under the premise that they do not cause secondary failure, which considerably improved the efficiency of the network function recovery. 

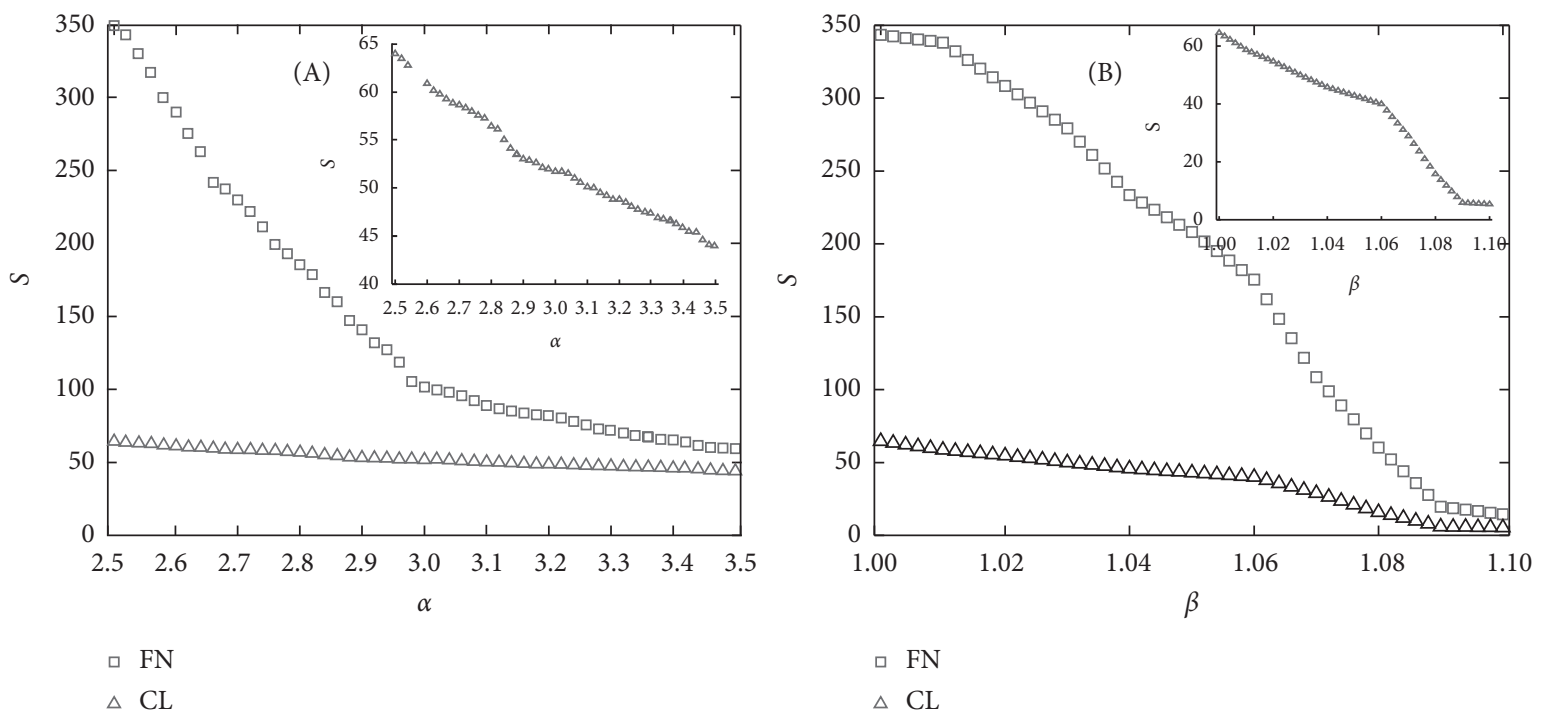

(a)
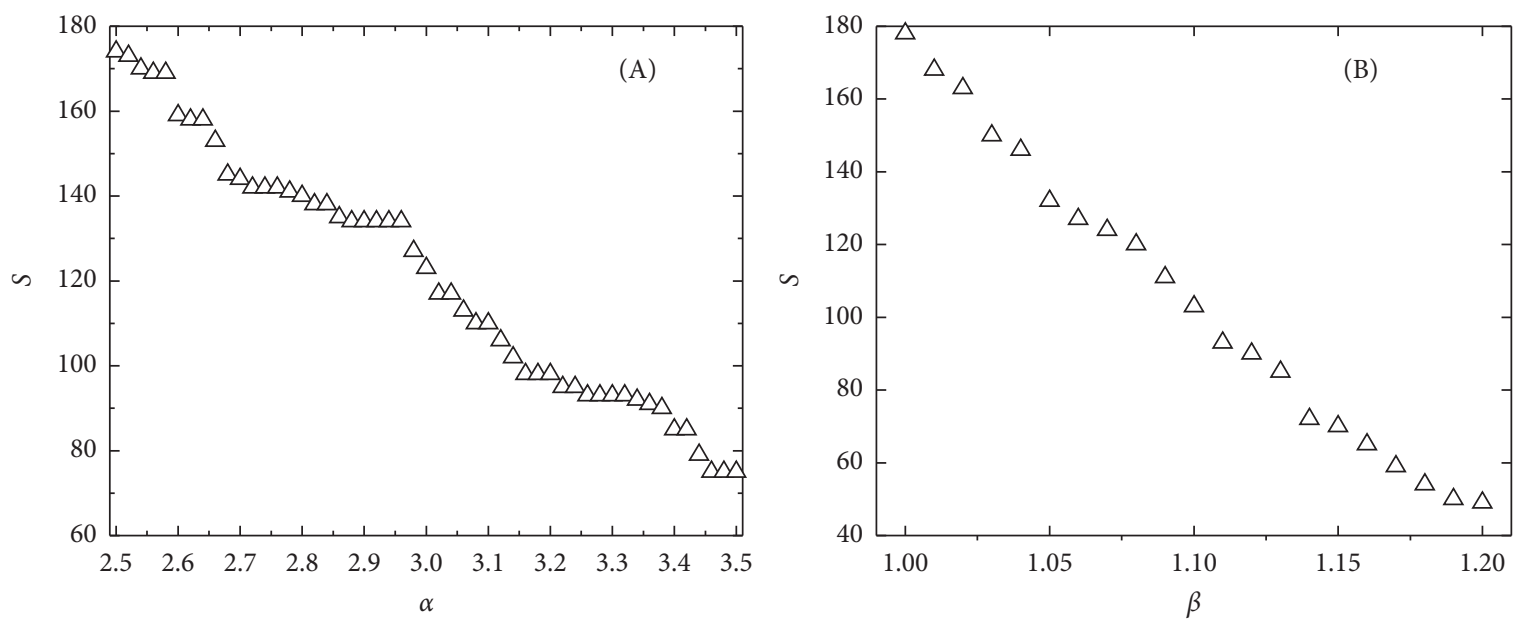

$\triangle \mathrm{CL}$

$\triangle \mathrm{CL}$

(b)

FIGURE 4: For (a) a scale-free network and (b) a scientific cooperation network, redundant edge characteristics in dynamic repair process vs tolerance coefficients (A) $\alpha$ (for fixed $\beta=1.0$ ) and (B) $\beta$ (for fixed $\alpha=3.0$ ).

\section{Robust Optimization}

Using a dynamic repair strategy, we can determine the redundant edges of inappropriate loads. When the energy transfer function on the redundant edge was cut off, the effect of the redistribution load on repaired nodes was reduced and the nondestructive repair was realized. The simulation results of Section 4 demonstrate the hazards of redundant edges. Thus, the two methods of eliminating redundant edges were proposed for optimizing the network.

5.1. Structure Optimization. Regardless of the influence of network parameters, the existence of redundant edges indicated that the network structure was unreasonable; therefore, the energy carried by some edges did not match the capacity of nodes connected to it. Cascade failure of the network can readily occur. Figure 5 presents the results of network robustness after structure optimization of the SCN. We filtered and removed redundant edges in the current network by repeating the operation until the network attained the optimal structure. Subsequently, we computed the scale of the cascading failure caused by failures of, or attacks on, a single node and recorded maximum values. By comparing the scale of failure nodes in the initial network (IFN) with that of the optimized network (OFN) we found that the robustness of the network was substantially improved. The results indicated that redundant edges considerably affected the cascading failure of the network, and the scale of network failure nodes decreased considerably. However, structural optimization was used to optimize the existing network structure by removing redundant edges. The drawback of the proposed strategy is that the load capacity of each node in the original network is also weakened. 


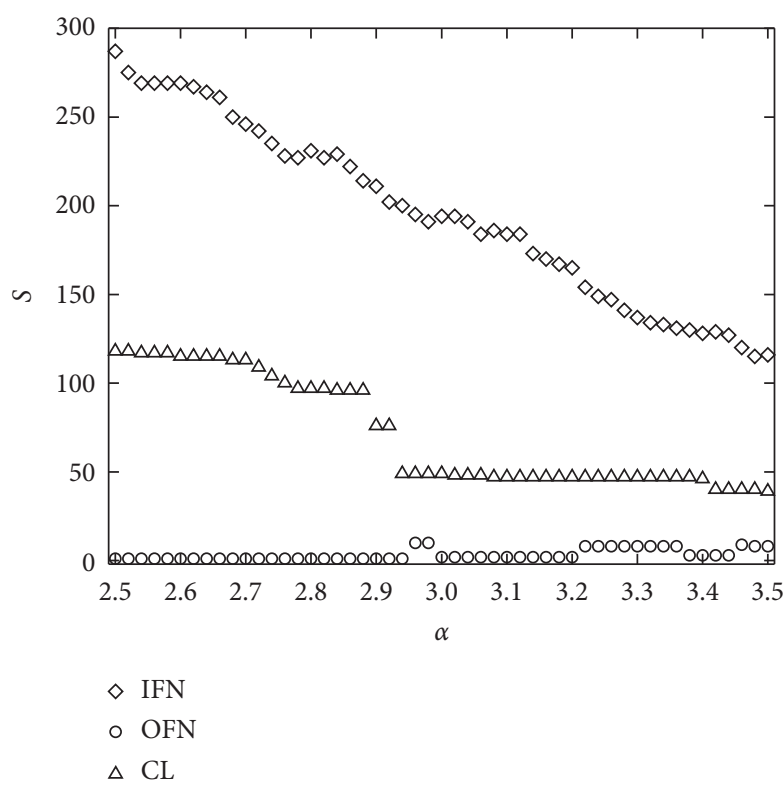

(a)

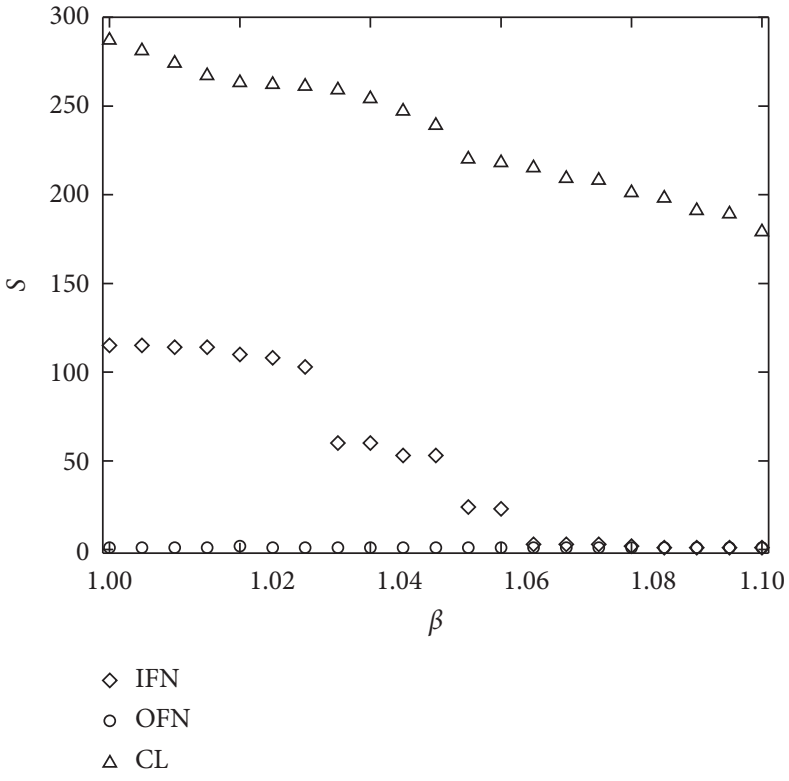

(b)

FIGURE 5: Scientific cooperation network, network robustness after structure optimization vs tolerance coefficients (a) $\alpha$ (fixed $\beta=1.0$ ) and (b) $\beta$ (fixed $\alpha=3.0$ ).

5.2. Capacity Optimization. Under the premise that the network structure was fixed, the existence of redundant edges indicated that some nodes in the network, whose redundant capacities were insufficient to withhold the energy shocks of adjacent failure nodes. A local perturbation easily spread to the entire network, and numerous hidden dangers undermined network security. The capacity optimization was based on our proposed algorithm to select the redundant edges and to calculate the transfer energy $e_{i j}$ and $e_{j i}$ on the redundant edge. When $e_{i j}<e_{j i}$, we increased the redundant capacity of node $j$ to offset the energy shocks of redundant edges, to prevent the node from having a risk of failure, and to enhance the robustness of the network. Figure 6 presents the results of the capacity optimization. The tolerance coefficient was set to $\alpha=3$ and $\beta=1$. The nodes with unreasonable capacity in the initial network were mostly nodes with a large redundant capacity, but this was not a strict standard. The redundant edge was selected according to the relationship between the redundant capacity of the node and transfer energy on the edge. The load of nodes and redundant capacity were positively correlated. The larger was the load of nodes, the higher was the transfer energy passing through the edge and more easily it became a redundant edge. However, when the redundant capacity of the node was reasonable, avoiding the formation of redundant edges was possible; the redundant edges can be removed by optimizing the redundant capacity. Figure 6 shows that 106 nodes were adjusted for network optimization; the average capacity of the optimized network after optimization was $C_{\mathrm{RN}}=8.2730$ compared with the average capacity of the original network $C_{\mathrm{ON}}=7.8232$. A larger gap caused the capacity setting of the original network to become more unreasonable. Capacity optimization involved finding

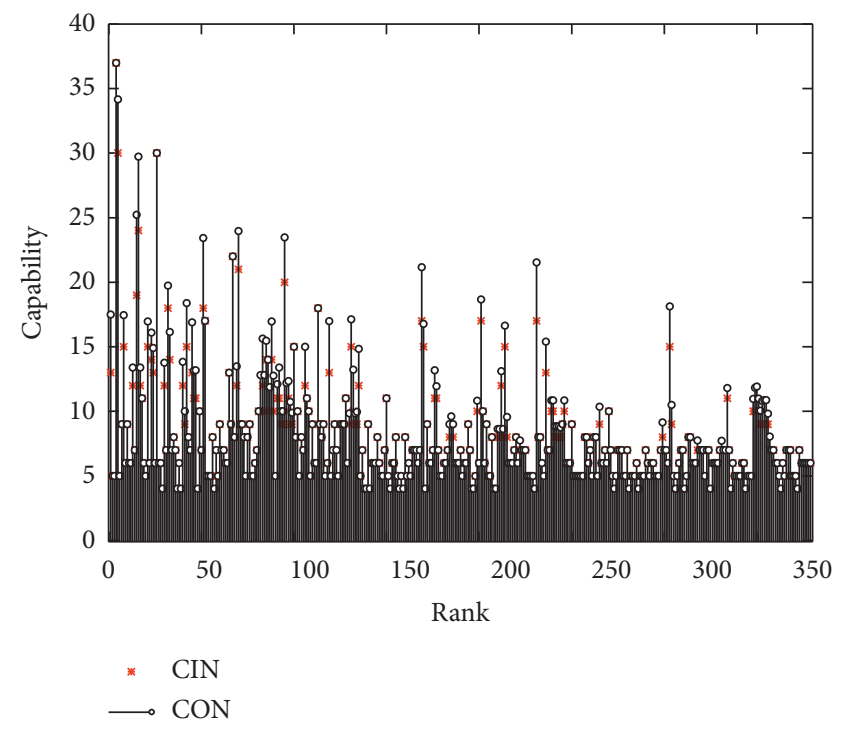

Figure 6: Capacity optimization results.

nodes with unreasonable capacity and optimizing the capacity of targeted nodes. The drawback of the proposed strategy is that the irrationality of the network structure is ignored.

\section{Conclusion}

Dynamic repair is complex and polytropic. Unreasonable repair is likely to cause the secondary failure of repaired nodes. However, appropriate repair strategies can prevent secondary failure and efficiently recover network functions. Considering the mutual influence of energy flow between 
nodes, a repair strategy in the dynamic model is proposed in this paper. The coupled structure in the failed network was the main reason for the secondary failure. The proposed algorithm can be used to filter the coupled structure in the network and to process the most destructive redundant edges in coupled structures, which effectively prevents the secondary failure of repaired nodes during repair. By rationally using the redundant capacity of repaired nodes to share some of the load of failure nodes, the recovery efficiency of the network function was considerably improved. In addition, the redundant edges were links with redundant energy in the network. Eliminating redundant edges can substantially improve the robustness of such networks.

\section{Data Availability}

The data used to support the findings of this study are available from the corresponding author upon request.

\section{Additional Points}

(1) A dynamic repair strategy is proposed to solve the secondary failure of the repaired nodes without any additional cost. (2) Structure optimization can eliminate edges with overload energy in the network structure. (3) Capacity optimization can help to design a reasonable capacity to enhance network robustness.

\section{Conflicts of Interest}

The authors declare that they have no conflicts of interest.

\section{Acknowledgments}

This work was supported by the Field Foundation of China (no. 61400020109).

\section{References}

[1] M. E. J. Newman, Networks: An Introduction, Oxford University Press, Oxford, UK, 2010.

[2] R. Albert, I. Albert, and G. L. Nakarado, "Structural vulnerability of the north American power grid," Physical Review E, vol. 69, no. 2, pp. 292-313, 2004.

[3] T. Ren, Y. F. Wang, M. M. Liu, and Y. J. Xu, "Analysis of robustness of urban bus network," Chinese Physics B, vol. 25, no. 2, Article ID 020101, 2016.

[4] R. Pastor-Satorras and A. Vespignani, Evolution and Structure of the Internet: A Statistical Physics Approach, Cambridge University Press, Cambridge, UK, 2004.

[5] H. R. Liu, M. R. Dong, R. R. Yin, and L. Han, "Cascading failure in the wireless sensor scale-free networks," Chiness Physics B, vol. 24, no. 5, Article ID 050506, 2015.

[6] J. Cai and S. Z. Yu, "An efficient management strategy for enhancing traffic capacity in scale-free networks," Acta Physica Sinica, vol. 62, no. 5, 2013, in Chinese.

[7] F. Radicchi, S. Fortunato, B. Markines, and A. Vespignani, "Diffusion of scientific credits and the ranking of scientists," Physical Review E, vol. 80, no. 5, Article ID 056103, 2009.

[8] P. Csermely, T. Korcsmáros, H. J. M. Kiss, G. London, and R. Nussinov, "Structure and dynamics of molecular networks: a novel paradigm of drug discovery," Pharmacology \& Therapeutics, vol. 138, no. 3, pp. 333-408, 2013.

[9] L. Y. Lü, D. B. Chen, X. L. Ren, Q.-M. Zhang, Y.-C. Zhang, and T. Zhou, "Vital nodes identification in complex networks," 2016, https://arxiv.org/abs/1607.01134.

[10] M. Xu, C. Y. Xu, and K. F. Cao, "Effect of degree correlations on controllability of undirected networks," Acta Physica Sinica, vol. 66, no. 2, Article ID 028901, 2017.

[11] Z. Su, L. Li, H. Peng, J. Kurths, and J. Xiao, "Robustness of interrelated traffic networks to cascading failure," Scientific Report, vol. 4, p. 5413, 2014.

[12] P. Holme, B. J. Kim, C. N. Yoon, and S. K. Han, "Attack vulnerability of complex networks," Physical Review E, vol. 65, no. 5, Article ID 056109, 2002.

[13] S. Boccaletti, V. Latora, Y. Moreno, M. Chavez, and D. Hwang, "Complex networks: structure and dynamics," Physics Reports, vol. 424, no. 4-5, pp. 175-308, 2006.

[14] R. Yang, W. X. Wang, Y. C. Lai, and G. Chen, "Optimal weighting scheme for suppressing cascades and traffic congestion in complex networks," Physical Review E, vol. 79, Article ID 026112, 2009.

[15] J. Wang, "Robustness of complex networks with the local protection strategy against cascading failures," Safety Science, vol. 53, pp. 219-225, 2013.

[16] L. P. Chi, R. Wang, H. Su et al., "Structural properties of US flight network," Chinese Physics Letters, vol. 20, no. 8, pp. 1393-1396, 2003.

[17] G. Chen, X. Wang, and X. Li, Introduction to Complex Networks: Models, Structures and Dynamics, Higher Education Press, Beijing, China, 2012.

[18] L. P. Chi, C. B. Yang, and X. Cai, "Stability of complex networks under the evolution of attack and repair," Chinese Physics Letters, vol. 23, no. 1, p. 263, 2006.

[19] R. S. Farr, J. L. Harer, and T. M. A. Fink, "Easily repairable networks: reconnecting nodes after damage," Physical Review Letters, vol. 113, no. 13, pp. 105-108, 2014.

[20] W. S. Sun and A. Zeng, "Target recovery in complex networks," The European Physical Journal B, vol. 90, no. 1, p. 10, 2017.

[21] Z. Li, Y. H. Guo, A. G. Xu, and Z. M. Hu, "Analysis of cascading dynamics in complex networks with an emergency recovery mechanism," Acta Physica Sinica, vol. 63, no. 15, Article ID 158901, 2014.

[22] A. Majdandzic, B. Podobnik, S. V. Buldyrev, D. Y. Kenett, S. Havlin, and H. Eugene Stanley, "Spontaneous recovery in dynamical networks," Nature Physics, vol. 10, no. 1, pp. 34-38, 2014.

[23] A. E. Motter and Y. C. Lai, "Cascade-based attacks on complex networks," Physical Review E, vol. 66, no. 6, Article ID 065102, 2002.

[24] J. Wang, L. Rong, L. Zhang, and Z. Zhang, "Attack vulnerability of scale-free networks due to cascading failures," Physica A: Statistical Mechanics and Its Applications, vol. 387, no. 26, pp. 6671-6678, 2008.

[25] C. Fu, Y. Wang, Y. Gao, and X. Wang, "Complex networks repair strategies: dynamic models," Physica A: Statistical Mechanics and Its Applications, vol. 482, pp. 401-406, 2017.

[26] A.-L. Barabási and R. Albert, "Emergence of scaling in random networks," Science, vol. 286, no. 5439, pp. 509-512, 1999.

[27] Wikipedia, 2014, https://github.com/gephi/gephi/wiki/ Datasets. 